\section{Spring and Fall Cabbage Cultivar Trials in Pennsylvania}

\author{
M.D. O rzolek, ${ }^{1}$ W.J . Lamont, ${ }^{2}$ \\ and L. O tjen ${ }^{3}$
}

\begin{abstract}
Additional IndeX words. Brasica oleraceae var. capitata
\end{abstract}

\begin{abstract}
Summary. T wenty-two cabbage cultivars were evaluated in the spring and 26 cabbage cultivars evaluated in the fall of 1997. The cultivars were evaluated for uniformity of maturity, marketable yield, percent cull, stem core length, and head firmness. In addition, three heads of each cultivar were tasted at harvest by the summer farm crew and responses noted on the data collection forms. The highest yielding cultivars were not necessarily the best performing ones evaluated in the trial. Average head weight was significantly different between spring and fall plantings. D ata from this trial suggests that multiple cultivars should be grown in Pennsylvania based on whether it is a spring or fall cabbage crop.
\end{abstract}

\footnotetext{
The cost of publishing this paper was defrayed in part by the payment of page charges. U nder postal regulations, this paper therefore must be hereby marked advertisement solely to indicate this fact.

1 Professor of vegetablecrops, D epartment of $\mathrm{H}$ orticulture, The Pennsylvania State U niversity, U niversity Park.

${ }^{2}$ Associate professor of vegetable crops, D epartment of $\mathrm{H}$ orticulture, The Pennsylvania State U niversity, U niversity Park.

${ }^{3}$ Project associate, D epartment of $\mathrm{H}$ orticulture, The Pennsylvania State U niversity, U niversity Park.
}

abbage, Brassica ol eraceae var. capitata L., has been a very important vegetable crop in Pennsylvania for the last 50 years. Cabbage has been primarily grown for the fresh wholesale and retail market with very little being used in the preprocessing or processing market. I n the last 10 years, the acreage of cabbage has dropped $\approx 40 \%$ in the state with most growers now growing cabbage for markets demanding very specific head size and quality. In addition, therehas been alarger array of cabbage maturities developed by seed companies to fit these various market segments (H owell, 1999; Scaife et al., 1998). From previous experience, cabbage cultivars maturing in less than $105 \mathrm{~d}$ will consistently produce uniform, tight heads in most of Pennsylvania (O rzolek et al., 1999).

\section{Materials and methods}

The cultivar trial was located at the $\mathrm{H}$ orticulture Research Farm, Russell E. Larson Research Center, Rock Springs, $\mathrm{Pa}$. C abbagetransplants were seeded on $17 \mathrm{M}$ arch 1997 in 200-cell Speedling trays (Speedling, Inc., Sun City, Fla.) for the spring trial and on 13 June 1997 in 128-cell Speedling trays for the fall trial. The soil type was a $\mathrm{H}$ agerstown silt loam with good drainage. O n 27 A pril 1997 before transplanting in the spring , $40 \mathrm{~N}-21.8 \mathrm{P}-83 \mathrm{~K} \mathrm{lb} /$ acre $(44.4 \mathrm{~N}$ 24.2P-93.3K kg.ha-1) was broadcast on the fall plowed wheat field and harrowed to incorporate the fertilizer. For the fall trial, an additional $12 \mathrm{lb} /$ acre of N was applied on 15 J uly 1997 followed by a harrow operation to incorporate the nitrogen. Cabbage cultivars were transplanted on $30 \mathrm{Apr}$. 1997 for the spring trial and on 21 July 1997 for the fall trial. The trial design wasa randomized completeblock with threereplications. Individual plotsconsisted of 25 plants spaced $1 \mathrm{ft}(30 \mathrm{~cm})$ apart between plants with $5 \mathrm{ft}$ (150 $\mathrm{cm}$ ) between rows in the spring trial. In the fall, the individual plots consisted of 12 plants spaced $2 \mathrm{ft}(60 \mathrm{~cm})$ in-the-row and $3 \mathrm{ft}(90 \mathrm{~cm})$ between rows since conditions are generally more optimum for plant growth and plants require more space to reach maximum head size.

The spring trial was drip irrigated using drip tape $[0.3 \mathrm{gal} / \mathrm{min}$ per 100 $\mathrm{ft}\left(1.1 \mathrm{~L} \cdot \mathrm{min}^{-1}\right.$ per $\left.30 \mathrm{~m}\right)$ ] (T-Tape International, San Diego, Calif.) hav- ing 12-inch $(30 \mathrm{~cm})$ orifice spacing on 2 M ay, 6 M ay, 26 J une, 3 July, 7 July, and 14 July 1997 . The fall cabbage trial wasirrigated only on 1 Aug. 1997. $\mathrm{N}$ o major diseases were encountered during cabbage production throughout the entire growing season. For weed control oxyfluorfen (a.i.) was applied postemergence at $1 \mathrm{pt} /$ acre (1.17 L $\cdot \mathrm{ha}^{-1}$ ) on $29 \mathrm{Apr}$. 1997. I nsects were controlled with applications of imidacloprid (a.i.) at $0.11 \mathrm{oz} / 100 \mathrm{ft}$ of row $(3 \mathrm{~g} / 30 \mathrm{~m}) 30 \mathrm{Apr}$. 1997, esfenvalerate (a.i.) at $8 \mathrm{fl} \mathrm{oz/}$ acre (585 $\mathrm{mL} \cdot \mathrm{ha}^{-1}$ ) on $2 \mathrm{M}$ ay 1997 and Bacillus thuringiensis kurstaki at $1.5 \mathrm{qt} /$ acre (3.51 L $\cdot \mathrm{ha}^{-1}$ ) on 13 June and 2 July 1997. For the fall cabbage trial, there was no herbicide application before transplanting. Weed control was accomplished by harrowing to prepare soil for transplanting and one cultivation. There were moderate populations of cabbage loopers (Trichoplusia ni $\mathrm{Hubner}$ ) and imported cabbage worms (PierisrapaeL.) during the fall cabbage cultivar trial requiring application of esfenvalerate (a.i.) at $8 \mathrm{oz} /$ acre (585 mL $\left.\cdot \mathrm{ha}^{-1}\right)$ on 25 July 1997 and also on 25 Aug. 1997, imidacloprid (a.i.) at $0.11 \mathrm{oz} / 100 \mathrm{ft}$ of row $(3 \mathrm{~g} /$ 30m) on 1 Aug. 1997 and Bacillus thuringiensis kurstaki at $1.5 \mathrm{qt} /$ acre (3.51 L.ha-1) on 25 Aug. 1997. H ead quality characteristics were collected from threeheads in the first replication only. Each of these heads were cut, measured, and tasted by the three person work crew.

Cabbage seeds were obtained from the following sources: Abbott and Cobb (Feasterville, Pa.), Asgrow Seed C o. (Seminis Vegetable Seeds, Saticoy, Calif.), H arris M oran Seed Co. (M odesto, Calif.), Petoseed (Seminis Vegetable Seeds, Saticoy, Calif.), N ovartis Seeds (formerly Rogers NK Seed Co., Boise, Idaho) and SakataSeed America(M organ H ill, Calif.).

\section{Results and discussion}

Spring 1997. All cabbage entries grew normally in the spring, but matured slowly due to the high temperatures experienced in July. 'D iscovery' (Petoseed/ Seminis), 'Augusta' (N ovartis), 'Atlantis' (Peto seed/ Seminis), 'E mblem' (Sakata), 'CB6' (N ovartis), 'Columbia' (Petoseed/ Seminis), 'CB2' (N ovartis), 'Blue Ridge' (Asgrow/ Seminis) and 'Blue Gem' (H arris M oran) were the top 
Table 1. Yield of cabbage cultivars grown in the spring trial at the Pennsylvania State U niversity, R ock Springs, Pa., 1997.

\begin{tabular}{|c|c|c|c|c|c|}
\hline \multirow[b]{2}{*}{ Cultivar } & \multirow[b]{2}{*}{ Seed source } & \multirow[b]{2}{*}{$\begin{array}{l}\text { Total yield } \\
\text { (tons/acre) }^{y}\end{array}$} & \multicolumn{2}{|c|}{ M arketable head } & \multirow[b]{2}{*}{$\begin{array}{c}\text { Proportion } \\
\text { of culls } \\
(\%)\end{array}$} \\
\hline & & & $\begin{array}{c}\text { Yield } \\
\text { (tons/acre) }\end{array}$ & $\begin{array}{c}\text { Avg } \\
\text { H ead wt } \\
\text { (Ib) }{ }^{x}\end{array}$ & \\
\hline D iscovery & Petoseed/ Seminis & 22.9 & 22.9 & 3.0 & 0 \\
\hline Augusta & N ovartis & 22.5 & 21.7 & 3.1 & 5 \\
\hline Atlantis & Petoseed/ Seminis & 19.0 & 18.8 & 3.2 & 12 \\
\hline Emblem & Sakata & 19.6 & 18.7 & 3.2 & 14 \\
\hline CB6 & N ovartis & 20.2 & 18.5 & 3.0 & 16 \\
\hline Columbia & Petoseed/ Seminis & 16.4 & 15.9 & 3.0 & 7 \\
\hline CB2 & N ovartis & 17.5 & 15.6 & 2.4 & 19 \\
\hline Blue Ridge & N ovartis & 17.2 & 15.1 & 2.6 & 22 \\
\hline Blue Gem & $\mathrm{H}$ arris $\mathrm{M}$ oran & 17.7 & 14.8 & 3.3 & 29 \\
\hline Cardinal & $\mathrm{H}$ arris $\mathrm{M}$ oran & 15.0 & 14.2 & 2.3 & 15 \\
\hline Bravo & $\mathrm{H}$ arris $\mathrm{M}$ oran & 17.3 & 14.1 & 3.0 & 34 \\
\hline Blue Thunder & $\mathrm{H}$ arris $\mathrm{M}$ oran & 15.4 & 13.4 & 2.4 & 25 \\
\hline Blue Bayou & Asgrow/ Seminis & 22.2 & 12.8 & 3.4 & 49 \\
\hline ХРН 15508 & Asgrow/ Seminis & 12.7 & 12.5 & 3.1 & 5 \\
\hline XPH 5957 & Asgrow/ Seminis & 15.6 & 12.4 & 2.5 & 32 \\
\hline ХРH 15516 & Asgrow/ Seminis & 15.5 & 11.7 & 2.5 & 34 \\
\hline ХРН 15513 & Asgrow/ Seminis & 15.2 & 11.3 & 2.4 & 35 \\
\hline ХРН 15514 & Asgrow/ Seminis & 20.4 & 10.5 & 3.1 & 48 \\
\hline XPH 15518 & Asgrow/ Seminis & 13.9 & 10.1 & 2.4 & 30 \\
\hline ХРН 15519 & Asgrow/ Seminis & 11.7 & 8.2 & 2.2 & 42 \\
\hline ХРH 15511 & Asgrow/ Seminis & 9.5 & 7.9 & 2.2 & 36 \\
\hline ХРH 15515 & Asgrow/ Seminis & 17.0 & 5.1 & 2.6 & 73 \\
\hline LSD $P \leq 0.05$ & & 7.6 & 8.6 & 0.7 & \\
\hline
\end{tabular}

${ }^{2}$ Asgrow Seed C 0. (Seminis V egetable Seeds), Saticoy, C alif.; H arrisM oran Seed C o., M odesto, Calif.; Petoseed (Seminis Vegetable Seeds), Saticoy, C alif.; N ovartis Seeds (fomerly Rogers N K Seed Co.), Boise, I daho and Sakata Seed America, M organ H ill, Calif.

y1.0 ton/ acre $=2.24 \mathrm{t} \cdot \mathrm{ha}^{-1}$.

$\times 1.0 \mathrm{lb}=0.45 \mathrm{~kg}$.

T able 2. H ead dimensions of cabbage cultivars grown in the spring cultivar trial at Pennsylvania State U niversity, R ock Springs, Pa., 1997.

\begin{tabular}{|c|c|c|c|c|c|c|}
\hline $\begin{array}{l}\text { H ead } \\
\text { shape }\end{array}$ & C ultivar & Color & $\begin{array}{c}\text { H ead } \\
\text { ht } \\
\text { (inch) }^{x}\end{array}$ & $\begin{array}{l}\text { H ead } \\
\text { width } \\
\text { (inch) }\end{array}$ & $\begin{array}{c}\text { Core } \\
\text { size }^{z} \\
\left(\text { inch }^{2}\right)^{w}\end{array}$ & $\begin{array}{c}\text { H ead } \\
\text { firmness }\end{array}$ \\
\hline \multirow[t]{3}{*}{ Flat/ Semi-flat: H ead height/ width ratio $\leq 0.8$} & ХРH 15514 & green & 5.0 & 6.2 & 21.0 & 1.8 \\
\hline & XPH 15518 & blue-green & 5.1 & 6.0 & 21.1 & 2.0 \\
\hline & ХРН 15519 & blue & 4.8 & 5.6 & 18.1 & 2.4 \\
\hline \multirow[t]{8}{*}{ Round: H ead height/ width ratio 0.81 to 0.97} & Blue Bayou & blue-green & 5.6 & 6.1 & 23.7 & 1.4 \\
\hline & ХРH 15513 & green & 5.4 & 5.8 & 18.2 & 1.6 \\
\hline & Bravo & blue-green & 5.9 & 6.2 & 20.9 & 1.8 \\
\hline & Blue Ridge & blue-green & 5.3 & 6.1 & 21.1 & 1.9 \\
\hline & Blue Gem & blue-green & 5.7 & 6.2 & 23.0 & 1.9 \\
\hline & ХРH 15516 & blue & 5.9 & 6.2 & 27.1 & 1.9 \\
\hline & ХРH 15515 & blue-green & 5.5 & 5.8 & 26.2 & 2.1 \\
\hline & Augusta & blue-green & 5.9 & 6.4 & 31.5 & 2.4 \\
\hline \multirow[t]{11}{*}{ O blong: $\mathrm{H}$ ead height / width ratio $\geq 0.98$} & ХРH 15508 & blue-green & 6.7 & 5.9 & 16.4 & 1.4 \\
\hline & D iscovery & green & 6.1 & 5.3 & 22.7 & 1.4 \\
\hline & CB 6 & green & 6.7 & 5.7 & 31.0 & 1.4 \\
\hline & Columbia & green & 6.1 & 5.6 & 24.6 & 1.6 \\
\hline & Atlantis & green & 6.4 & 5.3 & 17.2 & 1.6 \\
\hline & XPH 5957 & blue-green & 6.2 & 6.0 & 21.8 & 1.7 \\
\hline & CB 2 & blue & 5.8 & 5.7 & 19.3 & 1.8 \\
\hline & Cardinal & dark red & 6.4 & 6.0 & 25.5 & 1.8 \\
\hline & Blue Thunder & blue & 5.7 & 5.7 & 22.5 & 1.9 \\
\hline & XРH 15511 & green & 6.2 & 5.5 & 25.1 & 1.9 \\
\hline & Emblem & blue & 6.1 & 6.3 & 27.1 & 2.0 \\
\hline
\end{tabular}

${ }^{2}$ D etermined by multiplying core height by core width.

V Visually assessed on a scale of $1-5$, with 1 being tight and 5 being loose.

$\times 1.0$ inch $=2.54 \mathrm{~cm}$.

${ }^{\mathrm{w}} 1.0$ inch $^{2}=6.45 \mathrm{~cm}^{2}$. 
yielding cultivars based on marketable heads (Table 1 ). Of these cultivars, only 'CB6' from Novartis matured uniformly, although maturity occurred later than in the other cultivars. M ost of theearly-maturing cultivarsrequired many harvests, while the later-maturing cultivars sized more uniformly. 'Augusta' was the only round-headed cabbage cultivar with a large core of the top nine yielding cultivars, while the other cultivars were more oblong or tapered in shape(T able2). 'Atlantis' and ' $D$ iscovery' had the smallest internal cores of these five and produced very firm heads. ' $\mathrm{CB} 6$ ' also produced very firm heads but had a large internal core. 'C ardinal' was the only red cultivar available for the spring trial and produced auniform yield of good quality $2.3-\mathrm{lb}(1-\mathrm{kg})$ heads.

'Blue Bayou', 'Blue Gem, and 'B ravo' produced thelargest headsbut had the highest number of nonmarketable heads which lowered their marketable yields. 'XPH 15508' also had large head size, but matured nonuniformly, with many heads never reaching marketable size within the time span of this study.

Fall 1997. All cultivars matured uniformly during the fall evaluation. The total number of heads harvested was reduced in some cultivars due to a delay in maturity or head sizing. 'Blue Gem', 'E mblem', and 'Augusta' were the three top-yielding cultivars in the fall trial (Table 3). 'Blue Gem' had a medium/ large frame with red leaf margins, a medium-sized core, and an average head size of $6.6 \mathrm{lb}(3 \mathrm{~kg})$. 'E mblem' (Sakata) had a large frame and produced around head which was moderately loose, had an extremely large core, and an average head size of $6.6 \mathrm{lb}(3 \mathrm{~kg})$. 'Augusta' (N ovartis) produced a very firm round head averaging $6.3 \mathrm{lb}(2.9 \mathrm{~kg})$ with a mediumsized core on a medium-large framed plant. $\mathrm{H}$ ead dimensionsand head characteristics are presented for the fall cabbage cultivars in Table 4.
A new cultivar from Asgrow/ Seminis, 'XPH 15508', was notable for its excellent quality. It produced high yields of very sweet $6-\mathrm{lb}(2.7-\mathrm{kg})$ heads with very small cores and good firmness. Of the red cabbage cultivars evaluated in the fall, '831 YR' (Abbott and $\mathrm{Cobb}$ ) matured earliest but produced oblong or pointy heads with a very large internal core. '841' (Abbott and $(\mathrm{obb})$ had a smaller core and was more round but was less firm than '831 YR'.

O verall, total yield of most cabbage cultivars evaluated in the fall appeared similar to spring yields keeping in mind the fall trial had one-half the plant population of the spring trial. Yields of marketable cabbage weresignificantly higher in the fall trial compared to the spring trial mainly due to less favorable growing conditions experienced in the spring, specifically, below optimum temperature for cabbage development and head formation.

Table 3. Yield of cabbage cultivars grown in the fall cultivar trial at Pennsylvania State U niversity, R ock Springs, Pa., 1997.

\begin{tabular}{|c|c|c|c|}
\hline \multirow[b]{2}{*}{ Cultivar } & \multirow[b]{2}{*}{ Seed source } & \multicolumn{2}{|c|}{ T otal yield } \\
\hline & & $\begin{array}{c}\text { Yield } \\
\text { (tons/acre) }^{y}\end{array}$ & $\begin{array}{l}\text { Avg } \\
\text { wt } \\
\text { (lb) }\end{array}$ \\
\hline \multicolumn{4}{|l|}{ White cabbage } \\
\hline Blue Gem & $\mathrm{H}$ arris $\mathrm{M}$ oran & 23.3 & 6.6 \\
\hline Emblem & Sakata & 23.2 & 6.6 \\
\hline Augusta & N ovartis & 22.3 & 6.3 \\
\hline Blue Bayou & Asgrow/ Seminis & 20.5 & 5.8 \\
\hline XРH 15514 & Asgrow/ Seminis & 20.3 & 5.7 \\
\hline XPH 5957 & Asgrow/ Seminis & 19.8 & 5.9 \\
\hline Columbia & Petoseed/ Seminis & 19.5 & 5.5 \\
\hline ХРH 15515 & Asgrow/ Seminis & 18.8 & 5.3 \\
\hline XPH 15516 & Asgrow/ Seminis & 18.6 & 5.4 \\
\hline CB 6 & N ovartis & 16.7 & 5.3 \\
\hline CB 2 & N ovartis & 16.6 & 5.0 \\
\hline 780 & Abbott and Cobb & 16.4 & 4.9 \\
\hline XPH 15518 & Asgrow/ Seminis & 16.0 & 4.8 \\
\hline ХРН 15513 & Asgrow/ Seminis & 15.8 & 5.6 \\
\hline XPH 15511 & Asgrow/ Seminis & 13.7 & 4.4 \\
\hline 790 & Abbott and Cobb & 12.0 & 5.3 \\
\hline XPH 15519 & Asgrow/ Seminis & 11.0 & 4.3 \\
\hline \multicolumn{4}{|l|}{ Red cabbage } \\
\hline 831 YR & Abbott and Cobb & 17.1 & 4.8 \\
\hline 841 & Abbott and Cobb & 14.8 & 4.3 \\
\hline Cardinal & $\mathrm{H}$ arris $\mathrm{M}$ oran & 12.2 & 4.3 \\
\hline LSD $P \leq 0.05$ & & 6.6 & 1.3 \\
\hline
\end{tabular}

${ }^{\mathrm{Z}}$ Abbott and Cobb, Feasterville, Pa.; Asgrow Seed Co. (Seminis Vegetable Seeds), Saticoy, Calif.; H arris M oran Seed Co.,M odesto, Calif.; Petoseed (Seminis V egetable Seeds), Saticoy, Calif.; N ovartis Seeds, (formerly Rogers N K Seed Co.) Boise, I daho; and Sakata Seed America, M organ H ill, Calif. y1.0 ton/ acre $=2.24 \mathrm{t} \cdot \mathrm{ha}^{-1}$.

$\times 1.0 \mathrm{lb}=0.45 \mathrm{~kg}$. 
T able 4. H ead dimensions and characteristics of cabbage cultivars grown in the fall cultivar trial at Pennsylvania State U niversity, R ock Springs, Pa., 1997.

\begin{tabular}{|c|c|c|c|c|c|c|}
\hline $\begin{array}{l}\text { H ead } \\
\text { shape }\end{array}$ & C ultivar & Color & $\begin{array}{c}\text { H ead } \\
\text { ht } \\
\text { (inch) }^{x}\end{array}$ & $\begin{array}{l}\text { H ead } \\
\text { width } \\
\text { (inch) }\end{array}$ & $\begin{array}{c}\text { Core } \\
\text { size }^{z} \\
\left(\text { inch }^{2}\right)^{w}\end{array}$ & $\begin{array}{c}\text { H ead' } \\
\text { firmness }\end{array}$ \\
\hline \multirow[t]{4}{*}{ Flat/ semiflat: $\mathrm{H}$ ead height/ width ratio $\leq 0.8$} & Bravo & blue-green & 6.6 & 8.2 & 25.3 & 1.6 \\
\hline & Blue Ridge & blue-green & 6.1 & 7.6 & 19.7 & 1.6 \\
\hline & ХРH 15514 & green & 6.6 & 8.3 & 24.2 & 2.4 \\
\hline & XРH 15518 & blue-green & 6.1 & 8.1 & 18.7 & 2.9 \\
\hline \multirow[t]{18}{*}{ Round: H ead height/ width 0.81 to 0.97} & 780 & blue-green & 6.5 & 7.4 & 27.2 & 1.0 \\
\hline & Blue Gem & blue-green & 6.9 & 8.2 & 24.2 & 1.1 \\
\hline & Augusta & blue-green & 7.1 & 8.0 & 25.0 & 1.1 \\
\hline & D iscovery & green & 6.8 & 7.4 & 29.1 & 1.1 \\
\hline & Blue Bayou & blue-green & 6.5 & 8.1 & 26.6 & 1.2 \\
\hline & Columbia & green & 7.0 & 7.5 & 21.1 & 1.2 \\
\hline & Atlantis & green & 6.9 & 7.8 & 21.0 & 1.4 \\
\hline & XPH 5957 & blue-green & 6.9 & 7.8 & 22.2 & 1.4 \\
\hline & 841 & red & 6.3 & 6.9 & 20.0 & 1.6 \\
\hline & XPH 15508 & blue-green & 7.5 & 7.9 & 18.9 & 1.6 \\
\hline & ХРH 15516 & blue & 6.2 & 7.3 & 22.4 & 1.7 \\
\hline & Blue Thunder & blue & 6.6 & 7.7 & 26.0 & 1.7 \\
\hline & XРH 15511 & green & 6.6 & 7.3 & 27.0 & 1.9 \\
\hline & XPH 15513 & green & 6.5 & 7.5 & 21.1 & 2.2 \\
\hline & XPH 15515 & blue-green & 7.1 & 7.8 & 28.0 & 2.2 \\
\hline & ХРH 15519 & blue & 5.8 & 6.7 & 17.6 & 2.7 \\
\hline & Emblem & blue & 7.4 & 8.3 & 37.8 & 2.9 \\
\hline & 790 & blue & 6.4 & 7.8 & 24.5 & 3.3 \\
\hline \multirow[t]{4}{*}{ O blong: $\mathrm{H}$ ead height/ width $\geq 0.98$} & 831 YR & dark red & 6.8 & 6.8 & 33.9 & 1.3 \\
\hline & CB2 & blue & 7.1 & 7.0 & 22.3 & 1.4 \\
\hline & Cardinal & dark red & 7.1 & 7.2 & 25.5 & 2.9 \\
\hline & CB6 & green & 7.3 & 7.4 & 32.3 & 3.8 \\
\hline
\end{tabular}

${ }^{2}$ D etermined by multiplying core height by core width.

W isually assessed on a scale of 1 to 5 , with 1 being tight and 5 being loose.

$x 1.0$ inch $=2.54 \mathrm{~cm}$.

w1.0 inch ${ }^{2}=6.45 \mathrm{~cm}^{2}$.

O ur trial results suggest that for Pennsylvania, no single cultivar will perform best during the entire growing season, and that cultivars should be chosen based on seasonal performance. Best performing cultivars were chosen based on marketable yield as well as color, corelength, and head firmness. For spring, best performing cultivars in thistrial were; ' $D$ iscovery', 'Atlantis', 'Columbia', 'CB 2', and 'Blue Ridge'. For fall, best performing cultivars in this trial were; 'Blue Gem', 'Augusta', 'Bravo', 'Blue Bayou', and 'XPH 15508'.

\section{Literature cited}

H owell, J.C. 1999. Tips for successful production of cabbage. Proc. $1999 \mathrm{M}$ idAtlantic Fruit Veg. Conf., H ershey, Pa. p. 52.

O rzolek, M .D ., P.A. Ferretti, W.J . Lamont, Jr., A.A. M acN ab, J.M. H albrendt, S.J. Fleischer, Z. Smilowitz, and W.K. H ock. 1999. Pa. Comm. Veg. Prod. Recommendations. p. 177.

Scaife, K., W. Bash, and G. Wenneker. 1999. 1998 Cabbage cultivar evaluation for coleslaw. Proc. O hio Fruit Veg. Growers Conf., Toledo, O hio. p. 80-95. 